\title{
Small-x physics at the Large Hadron-electron Collider
}

\author{
Néstor Armesto*挤 \\ Departamento de Física de Partículas and IGFAE, Universidade de Santiago de Compostela \\ 15706 Santiago de Compostela, Galicia, Spain \\ E-mail: nestor.armestodusc.es
}

\begin{abstract}
I review the aspects of small- $x$ dynamics that can be addressed at the proposed Large Hadronelectron Collider ( $\mathrm{LHeC}$ ) at CERN, both in lepton-proton and in lepton-nucleus collisions. After an introduction, I discuss some selected results illustrating the capabilities of such machine to provide information on structure functions, inclusive and exclusive diffraction, and other observables of interest for constraining the high-energy dynamics of QCD. I focus on inclusive observables and on the capabilities with nuclear targets.
\end{abstract}

Light Cone 2010 - LC2010

June 14-18, 2010

Valencia, Spain

\footnotetext{
${ }^{*}$ Speaker.

${ }^{\dagger}$ I would like to express my gratitude to Max Klein and the co-conveners of the LHeC working group on Physics at High Parton Densities in ep and eA: Brian Cole, Paul Newman and Anna Stasto, as well as all the other participants in the elaboration of the Conceptual Design Report of the $\mathrm{LHeC}$, for a most pleasant collaboration. I also thank the organizers for the invitation to give this talk. This work has been supported by Ministerio de Ciencia e Innovación of Spain under projects FPA2008- 01177 and FPA2009-06867-E, by Xunta de Galicia (Consellería de Educación) and through grant PGIDIT07PXIB206126PR, and by the Spanish Consolider-Ingenio 2010 Programme CPAN (project CSD2007-00042).
} 


\section{Introduction}

Inclusive and diffractive data at small- $x$ from HERA and fixed-target experiments, can be described by different realizations of evolution equations within perturbative QCD: fixed-order perturbation theory (DGLAP), resummation schemes (BFKL/CCFM/CCSS/ABF) and non-linear evolution (BK). Unitarity of QCD as a quantum field theory implies that non-linear phenomena are unavoidable and saturation of partonic densities is expected to occur at high energies or small Bjorken- $x$, see Fig. 1. The Color Glass Condensate offers a non-perturbative but weak coupling realization of saturation ideas, by considering the slow modes as classical and allowing perturbative emissions from the fast partons, which leads via a renormalization group procedure to non-linear evolution equations, see [1] and references therein. The present discussion lies on the possibilities offered by existing data to distinguish among the different available schemes.

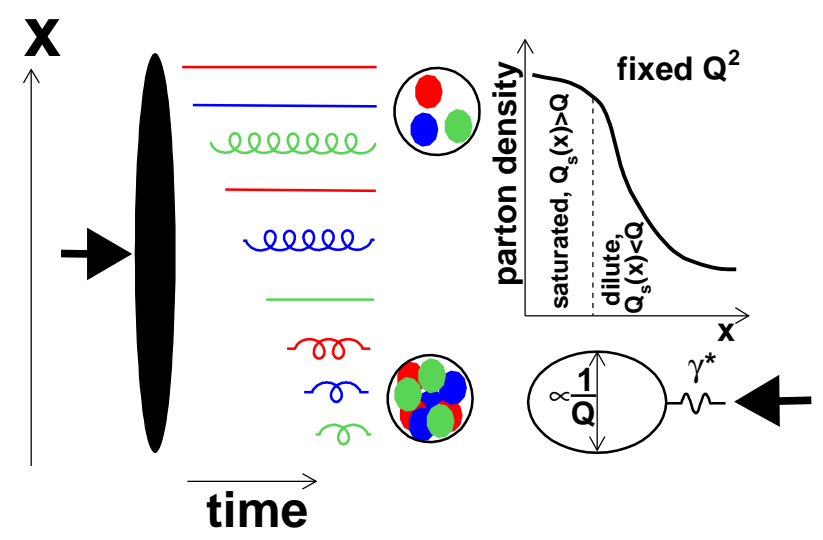

Figure 1: Illustration of the saturation ideas. The hadron is moving very fast to the right, and its wave function contains many partonic components. Apart from fast moving components it includes also slow moving partons which are characterized by the small fraction of its longitudinal momentum $x$. The photon with virtuality $Q^{2}$ is moving to the left and it constitutes a probe of the hadron wave function.

The Large Hadron-electron Collider ( $\mathrm{LHeC},[2,3])$ is an electron-proton/ion collider currently under design at CERN, which will collide $50 \div 150 \mathrm{GeV} e^{ \pm}$against the LHC beams, with a goal luminosity $10^{33} \mathrm{~cm}^{-2} \mathrm{~s}^{-1}$. Besides precision QCD and electro-weak studies, and searches for new physics, this machine should allow an unambiguous access to the novel regime of QCD in which unitarity constraints are at work - the dense region shown in Fig. 2. With the transition between the dilute region and the new phase being a density effect, a two-pronged approach will be pursued: either increasing $x$ at fixed mass number $A$ and $Q^{2}$, or increasing $A$ at fixed $x$ and $Q^{2}$.

From a more practical point of view, our knowledge of the gluon distribution at small $x$ both in proton and nuclei does not suffice for the required precision in predictions within collinear factorization at hadron colliders. Besides, in the $e$ A case and in the semihard region for particle production, collinear factorization is not expected to work and other factorization schemes have been proposed. Both aspects are of great importance for the study hadronic and nuclear collisions.

In this contribution I will review some aspects of small- $x$ physics which can be addressed with the $\mathrm{LHeC}$. Due to the limited space, I will focus on the possibilities with nuclear targets, and on 

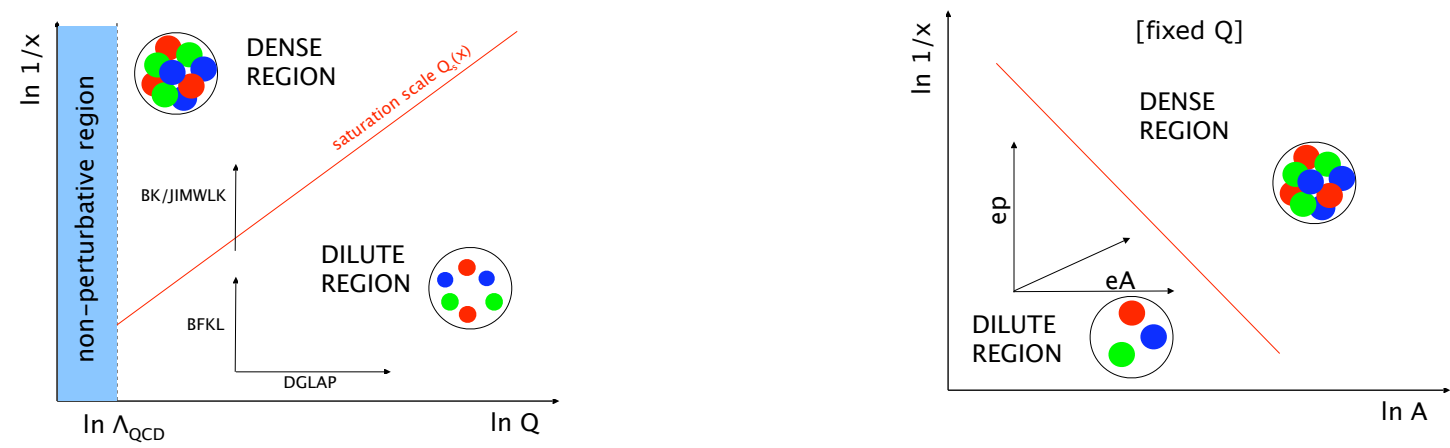

Figure 2: Sketch of the access to the dense partonic region where unitarity effects are essential, from the dilute one where linear evolution is valid. See the text for explanations.

inclusive observables. More information can be found in [3, 4], and in related work concerning the proposed Electron-Ion Collider at the US [5, 6].
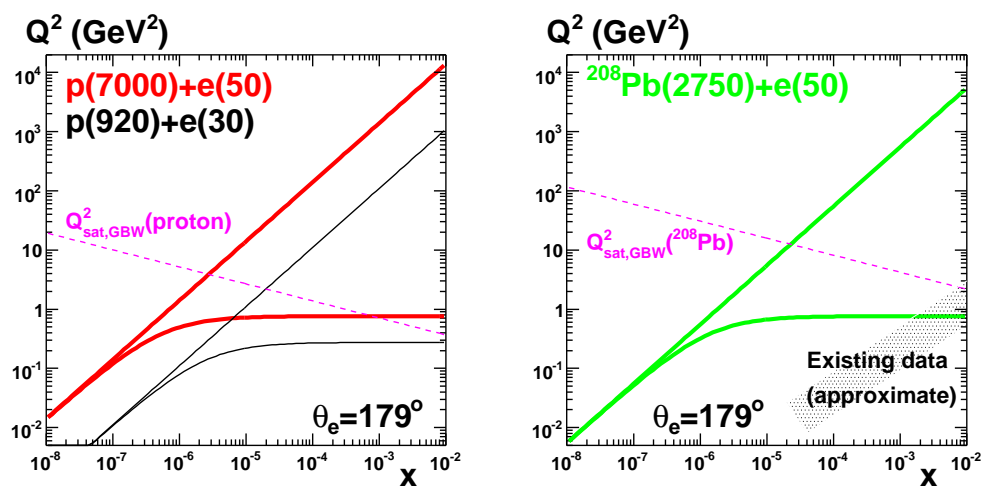

Figure 3: Region of the $Q^{2}-x$ plane that will be explored with the LHeC in $e p$ (left, red lines) and $e \mathrm{~Pb}$ (right, green lines), compared to those achievable at HERA (black lines on the left plot) and existing $e$ A experiments (shaded region on the right plot). The lines have been drawn for a 1-179 degree acceptance. An estimation of the saturation scale indicating the dilute-dense transition in both cases is shown (pink lines).

\section{Inclusive observables}

The $\mathrm{LHeC}$ will give access to a completely new region of the $Q^{2}-x$ plane, see Fig. 3. With this huge kinematical lever arm and the possibility to measure not only $F_{2}$ but also its flavor decomposition and $F_{L}$ (see Figs. 4 and 5 for examples of LHeC pseudodata on nuclear ratios of $F_{2}, F_{L}$ and $F_{2 c}$ ), the LHeC offers huge possibilities for:

- Constraining the parton densities in DGLAP analysis, both in $e p$ (see Rojo in [3]) and $e$ A (see [4] and Fig. 6). For this purpose, the combination of $F_{2}, F_{L}$ and $F_{2 c, b}$ appears to be very promising. As shown in Fig. 4 for $F_{2}$ and $F_{L}$ in the nuclear case, the expected uncertainty of data is much smaller than the spread of existing models. 

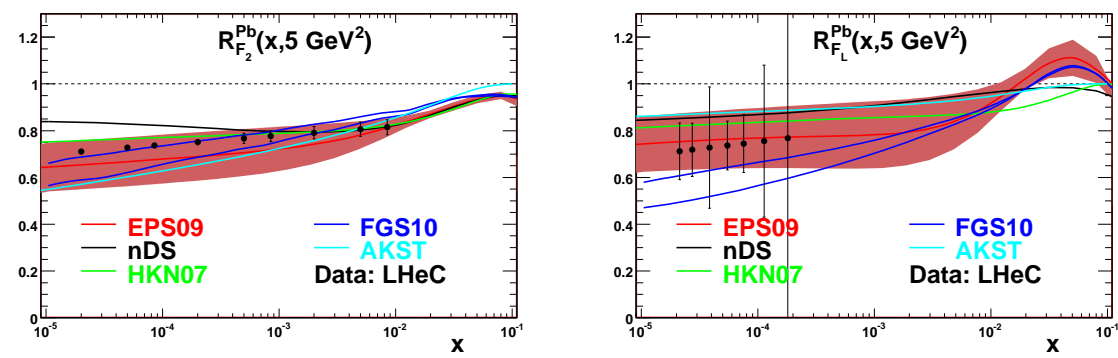

Figure 4: Predictions from different models [7] for the nuclear ratio $R_{F_{i}}^{\mathrm{Pb}}=F_{i}^{\mathrm{Pb}} /\left(208 F_{i}^{\mathrm{p}}\right), i=2, L$, at small $x$, see the legend on the plots. Circles with error bars are LHeC pseudodata.

- Disentangling fixed-order evolution schemes from resummation or non-linear ones, see [8]. For this purpose, the combination of data on $F_{2}$ and $F_{L}$ is required.

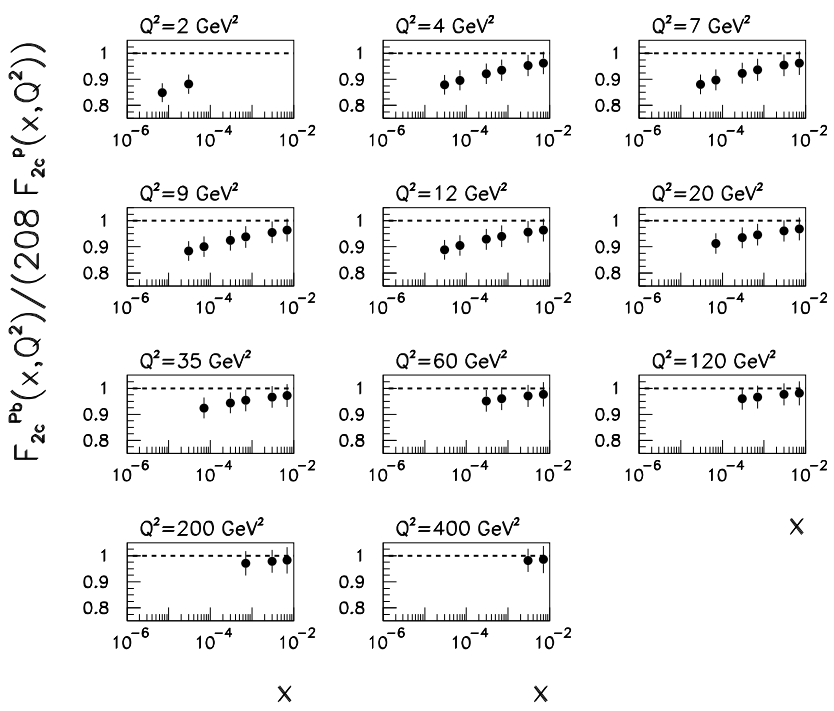

Figure 5: $\mathrm{LHeC}$ pseudodata for the nuclear ratio $R_{F_{2 c}}^{\mathrm{Pb}}=F_{2 c}^{\mathrm{Pb}} /\left(208 F_{2 c}^{\mathrm{p}}\right)$ at small $x$.

Work on including flavor decomposition in DGLAP analysis of $e \mathrm{p} \mathrm{LHeC} \mathrm{pseudodata,} \mathrm{and} F_{L}$ and flavor decomposition in $e \mathrm{~A}$, is under progress. Note that the nuclear effects on $F_{L}$ at small $x$ are unknown, which makes the extraction of $F_{2}$ at the smallest $x$ problematic in the nuclear case [9].

\section{Diffractive observables}

On diffraction in ep collisions [3, 4], the LHeC will explore a new domain of very low $\beta$ (e.g. down to a few times $10^{-4}$ for $Q^{2} \sim 4 \mathrm{GeV}^{2}$ at $x_{P}=0.003$, two orders of magnitude smaller than at HERA; see Fig. 7). Several aspects can be highlighted: 

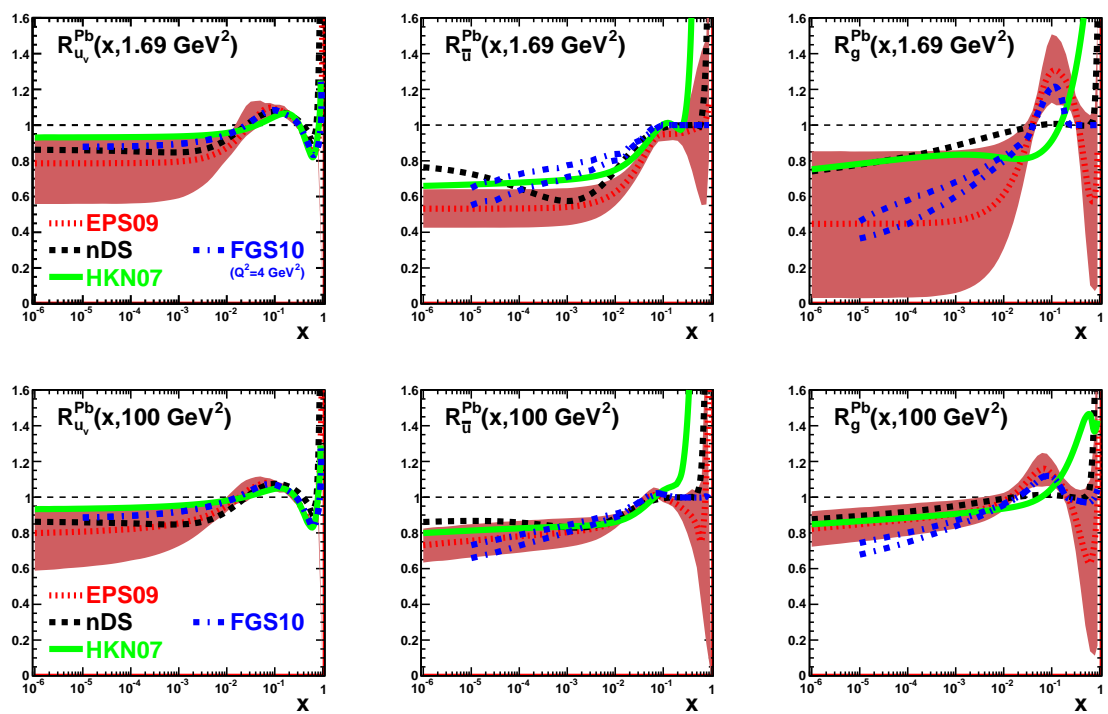

Figure 6: Ratio of parton densities in a bound proton in $\mathrm{Pb}$ over those in a free proton, for valence $u$ (left), $\bar{u}$ (middle) and $g$ (right), at $Q^{2}=1.69$ (top) and 100 (bottom) $\mathrm{GeV}^{2}$. Results from [7] (nDS, black dashed; HKN07, green solid; EPS09, red dotted; and FGS10, blue dashed-dotted - in this case the lowest $Q^{2}$ is 4 $\mathrm{GeV}^{2}$ and two lines are drawn reflecting the uncertainty in the predictions) are shown. The red band indicates in each case the uncertainties in the EPS09 analysis.

- It will give access with enough statistics to diffractive masses as large as $200 \mathrm{GeV}$, see Fig. 8, providing data to check models describing the transition from low to high masses, and to improve the determination of diffractive parton densities in DGLAP analysis.

- For elastic vector meson production or DVCS, a huge lever arm in $W$ will be explored (e.g. upto $\approx 1.2 \mathrm{TeV}$ for $E_{e}=50 \mathrm{GeV}$ ) with enough precision to disentangle linear evolution schemes from non-linear ones. The differential spectrum in $t$ will also be accessible up to $t \sim-2 \mathrm{GeV}^{2}$.

- Gribov's relation between diffraction in $e p$ and nuclear shadowing will be checked in a single experimental setup (see e.g. the FGS10 and AKST models in [7] and Fig. 4).

In $e \mathrm{~A}$, the perspectives for the reach in kinematics are very similar to those in $e p$ - with the difference that for nuclei information on diffraction at small $x$ (see Armesto in [3]) does not exist at all. The experimental challenge in separating inclusive diffraction $\left(e+\mathrm{A} \rightarrow e+X+X^{\prime}\right.$ with a rapidity gap) from coherent $(e+\mathrm{A} \rightarrow e+X+\mathrm{A})$ and incoherent $(e+\mathrm{A} \rightarrow e+X+Z \mathrm{p}+[A-Z] \mathrm{n})$ is under study.

\section{Final states}

Besides the study of jets for the determination of $\alpha_{s}$ (see Behnke in [3]), the LHeC will offer huge possibilities for:

- Clarifying the dynamics of hadronization, through testing the parton/hadron energy loss mechanism in SIDIS by introducing a piece of colored material - the nucleus - which would modify its pattern (length/nuclear size, chemical composition; see Brooks in [6]). Energies as high as $10^{5}$ 
Diffractive Kinematics at $x_{1 \mathrm{P}}=0.01$

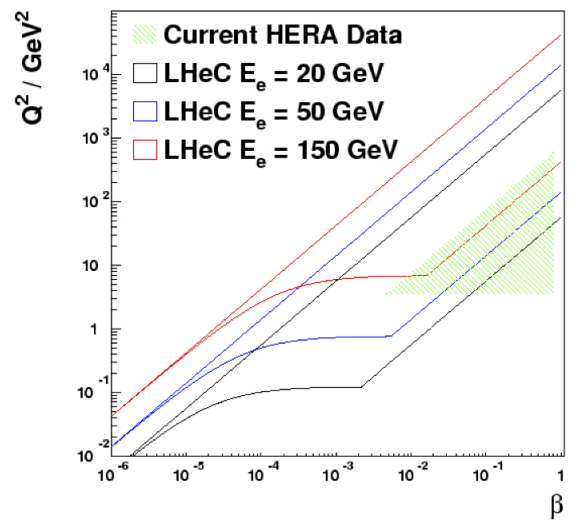

Diffractive Kinematics at $x_{\mathrm{IP}}=0.0001$

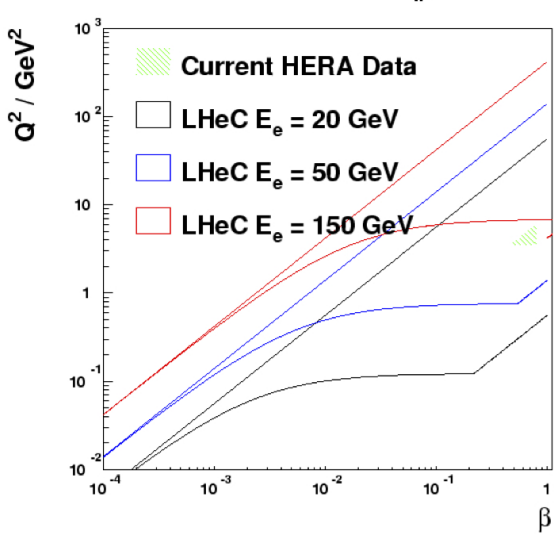

Figure 7: Kinematic range of LHeC in $Q^{2}$ and $\beta$ for different electron energies $E_{e}=20,50,150 \mathrm{GeV}$. Left plot: $x_{P}=0.01$, right plot: $x_{P}=0.0001 .1^{\circ}$ acceptance of the detector is assumed.

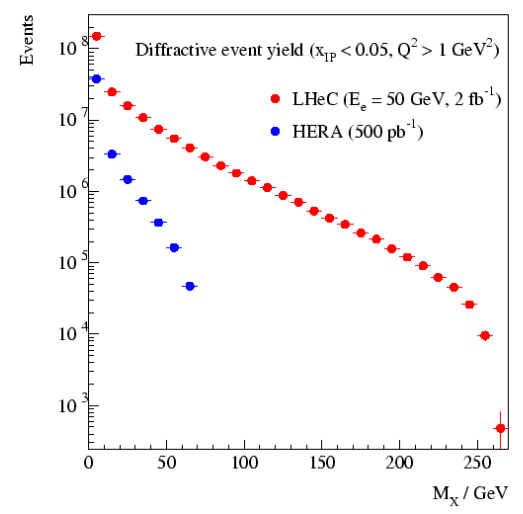

Figure 8: Diffractive event yield versus the mass of the diffractive state: comparison between HERA and the $\mathrm{LHeC}$.

$\mathrm{GeV}$ in the rest frame of the nucleus will be accessible and the transition from low to high energies (with hadronization expected to occur inside or outside the nucleus respectively) could be studied.

- Establishing the dynamics of QCD radiation though the measurement of forward jets in DIS (see Kutak in [3]).

As an example of the abundant yield of high-energy probes of the nuclear medium, the inclusive jet rates in photoproduction have been computed using the code in [10], for an electron beam of $50 \mathrm{GeV}$ colliding with the LHC beams, and assuming in the nuclear case the same integrated luminosity per nucleon of $2 \mathrm{fb}^{-1}$ as for $e$ p. Only jets with $E_{T \text { jet }}>20 \mathrm{GeV}$ are considered, and for the distribution in $E_{T \text { jet }}$ the pseudorapidity acceptance is $\left|\eta_{\text {jet }}\right|<3.1$, corresponding to $5^{\circ}<\theta_{\text {jet }}<175^{\circ}$ angular acceptance. The choices in the code are: (i) For the Weizsäcker-Williams distribution of the electron, the standard option in [10]; (ii) For the photon parton densities, GRV-HO [11]; (iii) For the proton parton densities, CTEQ6.1M [12]; (iv) For the nuclear modification of nucleon parton densities, EPS09 [7]; (v) For the renormalization and factorization scales, $\mu_{R}=\mu_{F}=\sum_{\text {jets }} E_{T \text { jet }} / 2$; and (vi) For the jet definition algorithm, inclusive $k_{T}$ [13] with $D=1$. The statistical uncertainty 
in the computation (i.e. in the Monte Carlo integration) is smaller than $10 \%$ for all shown results, being usually much smaller and only of that order for the largest $E_{T \text { jet }}$. No attempt has been done to estimate the uncertainties due to different choices of Weizsäcker-Williams distribution of photons in the electron, photon or proton parton densities, scales or jet definitions (see [14, 15] for such considerations at HERA). Nor the eventual problems of background subtraction, experimental efficiencies in jet reconstruction or energy calibration, have been addressed. The only studied uncertainty studied is that due to the uncertainties in the nuclear parton densities, extracted in EPS09 [7] using the Hessian method, see that reference for details.

The results are shown in Fig. 9. The main observations to be done are: (a) Rates around $10^{3}$ jets per $\mathrm{GeV}$ are expected with $E_{T j e t} \sim 95(80) \mathrm{GeV}$ in $e \mathrm{p}(e \mathrm{~Pb})$, for $\left|\eta_{j e t}\right|<3.1$ and the considered integrated luminosity of $2 \mathrm{fb}^{-1}$ per nucleon; (b) The effects of the nuclear modification of parton densities and their uncertainties are smaller than $10 \%$; and (c) The two-peak structure in the $\eta_{\text {jet }}{ }^{-}$ plot results from the sum of the direct plus resolved contributions, each of them with a single maximum but located in opposite hemispheres: positive $\eta_{\text {jet }}$ (photon side) for direct, negative $\eta_{j e t}$ (nucleon side) for resolved.
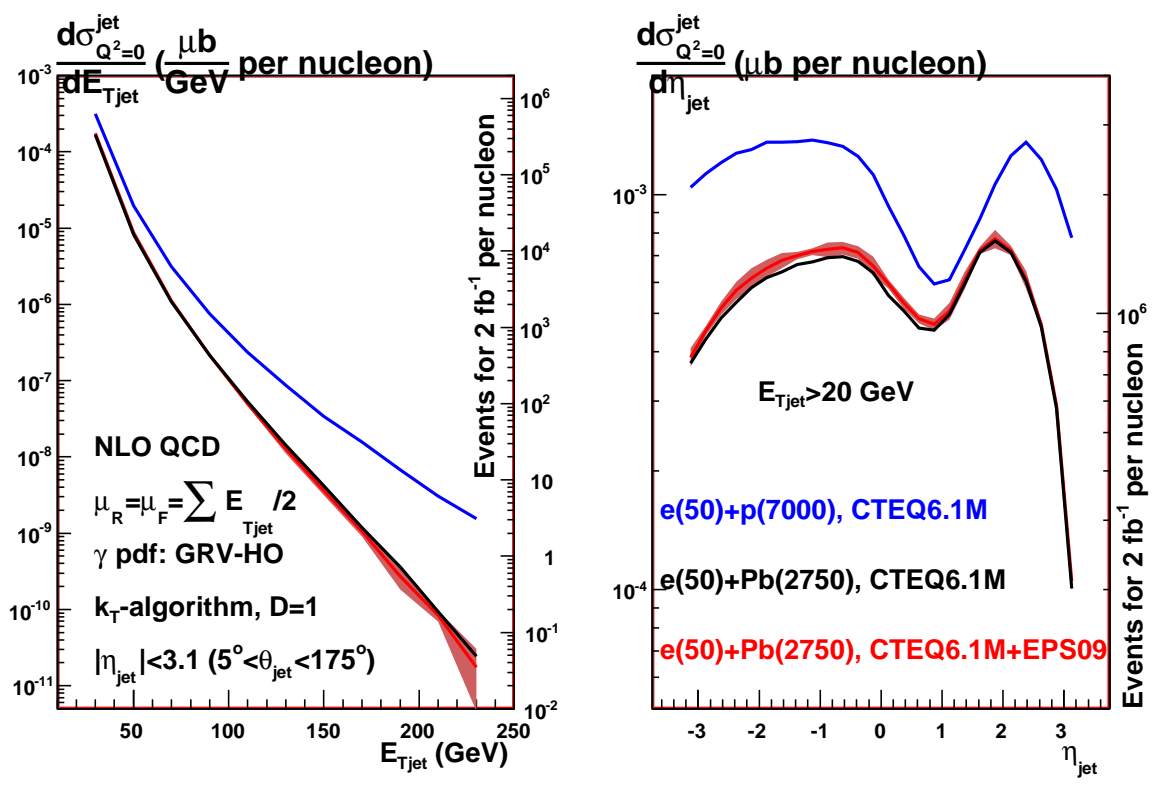

Figure 9: Results for the inclusive jet distribution in photoproduction versus $E_{T j e t}$ (plot on the left) and $\eta_{j e t}$ (plot on the right) for $e(50)+\mathrm{p}(7000)$ (blue lines), $e(50)+\mathrm{Pb}(2750)$ without nuclear modification of parton densities (black lines), and $e(50)+\mathrm{Pb}(2750)$ with EPS09 nuclear modification of parton densities (red lines for the central value and bands for the uncertainty coming from the nuclear modification of parton densities). See the text and the legends on the plots for information about choices in the calculation and kinematical cuts. In both plots, the axis on the left corresponds to the cross section in $\mu \mathrm{b}$, while the axis on the right provides the number of jets to be observed for an integrated luminosity of $2 \mathrm{fb}^{-1}$ per nucleon, per unit of $E_{T \text { jet }}\left(\eta_{j e t}\right)$ in the plot on the left (right).

Finally, studies of hadronic final states through Monte Carlo simulation using DPMJET-III [16] for the purpose of detector design, are under way [3]. 
Summarizing, I have presented some of the observables and opportunities at small $x$, whose measurement at the $\mathrm{LHeC}$ will offer most valuable information to clarify the high-energy behavior of the strong interaction. Work on all these aspects is in progress, with the aim of producing a Conceptual Design Report before the end of 2010.

\section{References}

[1] F. Gelis, E. Iancu, J. Jalilian-Marian and R. Venugopalan, arXiv:1002.0333 [hep-ph].

[2] M. Klein et al., Proceedings of 11th European Particle Accelerator Conference (EPAC 08), Magazzini del Cotone, Genoa, Italy, 23-27 June 2008, preprint WEOAG01; http: / / cern. ch / I hec.

[3] For recent information, see the LHeC talks at DIS 2010:

N. Armesto,

http: //indico. cern. ch/contributionDisplay . py? contrib Id=305\& conf Id=86184;

P. Kostka, contribId=293; O. Behnke, $i b$. contribId=333;

K. Kutak, $i b$. contribId=312; J. Rojo, ib. contribId=306;

J. Jowett, $i b$. contribId=290; S. Levonian, $i b$. contribId=297;

G. Azuelos, $i b$. contribId=45; U. Klein, $i b$. contribId $=44$.

[4] P. Newman, in M. Deile et al., arXiv:1002.3527 [hep-ph].

[5] The Electron Ion Collider Working Group Collaboration, C. Aidala et al., A High Luminosity, High Energy Electron Ion Collider; http: //web. mit. edu/eicc/.

[6] For recent information, see the EIC talks at DIS 2010:

A. Deshpande,

http: //indico. cern. ch/contributionDisplay . py? contribId=321\&conf Id=86184;

W. Brooks, $i b$. contribId=334; M. Lamont, $i b$. contribId=308.

[7] D. de Florian and R. Sassot, Phys. Rev. D 69 (2004) 074028;

M. Hirai, S. Kumano and T. H. Nagai, Phys. Rev. C 76 (2007) 065207;

K. J. Eskola, H. Paukkunen and C. A. Salgado, JHEP 0904 (2009) 065;

V. Guzey and M. Strikman, Phys. Lett. B 687 (2010) 167;

N. Armesto, A. B. Kaidalov, C. A. Salgado and K. Tywoniuk, Eur. Phys. J. C 68 (2010) 447.

[8] J. Rojo and F. Caola, arXiv:0906.2079 [hep-ph]; F. Caola, S. Forte and J. Rojo, Phys. Lett. B 686 (2010) 127.

[9] N. Armesto, H. Paukkunen, C. A. Salgado and K. Tywoniuk, arXiv:1005.2035 [hep-ph].

[10] S. Frixione, Z. Kunszt and A. Signer, Nucl. Phys. B 467 (1996) 399; S. Frixione, Nucl. Phys. B 507 (1997) 295; http://www.ge.infn.it/ ridolfi/dijet/.

[11] M. Gluck, E. Reya and A. Vogt, Phys. Rev. D 45 (1992) 3986.

[12] D. Stump, J. Huston, J. Pumplin, W. K. Tung, H. L. Lai, S. Kuhlmann and J. F. Owens, JHEP 0310 (2003) 046.

[13] S. D. Ellis and D. E. Soper, Phys. Rev. D 48 (1993) 3160.

[14] C. Adloff et al. [H1 Collaboration], Eur. Phys. J. C 29 (2003) 497.

[15] S. Frixione and G. Ridolfi, Nucl. Phys. B 507 (1997) 315.

[16] R. Engel, J. Ranft and S. Roesler, Phys. Rev. D 52 (1995) 1459; ib. 55 (1997) 6957; ib. 57 (1998) 2889. 